\title{
ESTUDO DO COMPORTAMENTO DA EFICIÊNCIA DE DEPOSIÇÃO DA LIGA Co-W UTILIZANDO PLANEJAMENTO EXPERIMENTAL.
}

\author{
M. B. PORTO ${ }^{1 *}$, M. G. A. VIEIRA ${ }^{1}$, M. G. C. $\operatorname{SILVA}^{1}$, A. F. ALMEIDA NETO ${ }^{1}$ \\ ${ }^{1}$ Universidade Estadual de Campinas, Faculdade de Engenharia Química, \\ Departamento de Desenvolvimento de Processos e Produtos. \\ *e-mail: maribporto@hotmail.com
}

\begin{abstract}
RESUMO
Uma das formas que mais contribui para o desgaste dos metais é sem dúvida a corrosão. O controle e estudo para minimizar os efeitos causados por esse processo são essenciais. A eletrodeposição é um dos métodos mais importantes de deposição metálica para a produção comercial de películas protetoras. O desenvolvimento de banhos estáveis com adequados níveis de $\mathrm{pH}$, temperatura, concentração de reagentes e densidade de corrente são de fundamental importância para obter depósitos de boa qualidade e resistente a corrosão. Este trabalho estudou o efeito da concentração de sulfato de cobalto, temperatura do banho e densidade de corrente para obtenção de deposito da liga Co-W. Para o estudo proposto, dentro da faixa de variáveis utilizadas, a maior porcentagem de deposição encontrada foi de $86,61 \%$, utilizando concentração de sulfato de cobalto $0,3 \mathrm{~mol} / \mathrm{L}$, temperatura de $25^{\circ} \mathrm{C}$ e densidade de corrente igual a $10 \mathrm{~mA} / \mathrm{cm}^{2}$. As ligas obtidas apresentaram boa aderência e brilho metálico.
\end{abstract}

\section{INTRODUÇÃO}

A corrosão pode incidir sobre diversos tipos de materiais, este fenômeno aparece frequentemente e nas mais variadas atividades, como por exemplo, nas indústrias química, petroquímica, naval, de construção civil, entre outras (GENTIL, 1996). O processo de corrosão produz alterações prejudiciais e indesejáveis nos elementos estruturais como a deterioração do material.

O produto da corrosão é um elemento diferente do material original, com menor resistência mecânica, elasticidade, ductilidade e estética (AZEVEDO, 2010). A utilização de revestimentos protetores é um dos principais métodos de proteção à corrosão. Dentre estes revestimentos, os metálicos são de uma grande importância industrial, por apresentarem boas propriedades mecânicas e físicas, como: alta dureza, resistência ao impacto, ductibilidade e resistência à corrosão (KOTZ, 2005).

As camadas protetoras metálicas são aplicadas por imersão a quente, cementação, revestimento mecânico, eletrodeposição, condensação de vapor do metal e pulverização do metal. Como consequência do desenvolvimento dos geradores de corrente contínua a prática da eletrodeposição tornouse o processo mais utilizado para a proteção dos metais. Além disso, a eletrodeposição tem provado ser um método válido para se preparar materiais magnéticos, onde as ligas de níquel-ferro e de cobalto são bastante empregadas para esse proposito (WOLYNEC, 1998).

A eletrodeposição de ligas metálicas é teoricamente fundamentada na Lei de Ohm e nas Leis de Faraday. A reação eletroquímica consiste na troca de elétrons entre duas semirreações de oxirredução. A redução de 
certos componentes presentes no eletrólito ocorre em decorrência da passagem de uma corrente elétrica pelo eletrolítico, no entanto a reação eletroquímica processa-se exclusivamente na interface eletrodoeletrólito (DELAHAY, 1965).

$\mathrm{Na}$ investigação do processo de eletrodeposição é preciso o estudo das soluções que serão utilizadas para desenvolver o banho. A determinação da composição do banho eletrolítico exige um conhecimento prático da eletroquímica dos elementos que constituirão a liga, da solubilidade de seus sais e da química de seus complexos.

A operação satisfatória de um banho de deposição requer o controle das variáveis de composição: natureza dos complexos, $\mathrm{pH}$, razão molar entre os íons metálicos e das variáveis de operação: temperatura, densidade de corrente e agitação (BRENNER, 1964). Estudar o processo de eletrodeposição de uma determinada liga envolve, além do conhecimento de diversas áreas, um critério para escolha do material que irá compor esta liga. $O$ tungstênio apresenta características incomuns em relação à maioria dos metais. Este metal é atóxico em ambientes aquáticos, não carcinogênico e possui alto ponto de fusão $\left(3.410{ }^{\circ} \mathrm{C}\right)$, o que torna impossível depositá-lo termicamente sobre a superfície de qualquer outro metal, apresenta ainda baixo coeficiente de dilatação linear térmica (4,3×10-6 $\left.{ }^{\circ} \mathrm{C}-1\right)$, alta condutividade térmica $\left(0,487 \mathrm{cal} / \mathrm{cm}{ }^{\circ} \mathrm{C}\right)$, e é um dos metais mais densos $\left(19,3 \mathrm{~g} / \mathrm{cm}^{3}\right)$. O tungstênio possui excelentes propriedades mecânicas, sendo capaz de conservar sua dureza mesmo em altas temperaturas, tem a mais alta resistência à tração $\left(410 \mathrm{Kg} / \mathrm{mm}^{2}\right)$ e é muito resistente à corrosão, não sendo atacado por nenhum ácido mineral simples na temperatura ambiente (GENTIL, 1996).

Entretanto, esse metal não é depositado sozinho em meio aquoso, mas na presença de elementos do grupo $\mathrm{Fe}$ ( $\mathrm{Fe}$, $\mathrm{Co}$ e $\mathrm{Ni}$ ), sendo esta eletrodeposição classificada por Brenner (1964) como induzida. Em relação ao mecanismo de eletrodeposição das ligas M-W $(\mathrm{M}=\mathrm{Ni}, \mathrm{Co}$ ou $\mathrm{Fe})$, a proposição mais aceita supõe que a eletrodeposição do tungstênio ocorre em decorrência da formação de um complexo precursor, como, por exemplo $\left[(\mathrm{Ni})\left(\mathrm{WO}_{4}\right)(\mathrm{Cit})(\mathrm{H})\right]^{2-} \quad$ ou $\left[(\mathrm{Ni})\left(\mathrm{WO}_{4}\right)_{2}\left(\mathrm{H}_{2}\right)(\mathrm{Cit})\right]^{3-}$, formados de acordo com as Equações 1 e 2 (BROOMAN, 2004):

$$
\begin{aligned}
& {\left[(\mathrm{Ni})\left(\mathrm{WO}_{4}\right)(\mathrm{Cit})(\mathrm{H})\right]^{2-}+\left[\left(\mathrm{WO}_{4}\right)(\mathrm{Cit})(\mathrm{H})\right]^{4-} \rightarrow} \\
& {\left[(\mathrm{Ni})\left(\mathrm{WO}_{4}\right)_{2}\left(\mathrm{H}_{2}\right)(\mathrm{Cit})\right]^{3-}+\mathrm{Cit}^{3-}} \\
& {[(\mathrm{Ni})(\mathrm{Cit})]^{-2}+\left[\left(\mathrm{WO}_{4}\right)(\mathrm{Cit})(\mathrm{H})\right]^{4-} \rightarrow} \\
& {\left[(\mathrm{Ni})\left(\mathrm{WO}_{4}\right)(\mathrm{Cit})(\mathrm{H})\right]^{2-}+\mathrm{Cit}^{3-}}
\end{aligned}
$$

Da análise de dados desta liga, é possível obter uma melhor compreensão da influência das características dos constituintes dos eletrólitos e das variáveis de operação sobre as características da liga obtida e sobre o próprio processo de eletrodeposição.

Este trabalho teve como objetivo sintetizar ligas de $\mathrm{Co}-\mathrm{W}$ de boa qualidade pela técnica da eletrodeposição com o intuito de mitigar o efeito da corrosão, avalianndo a influência da variação da concentração de sulfato de cobalto, densidade de corrente e temperatura do banho na eficiência de deposição.

\section{METODOLOGIA}

\subsection{Eletrodeposição}

$\mathrm{O}$ banho eletroquímico utilizado foi composto por tungstato de sódio, $\mathrm{Na}_{2} \mathrm{WO}_{4} 0,3$ $\mathrm{mol} / \mathrm{L}$, sulfato de cobalto, $\mathrm{CoSO}_{4}$, como fonte do cobalto e citrato de amônio, $\left(\mathrm{NH}_{4}\right)_{2} \mathrm{C}_{6} \mathrm{H}_{6} \mathrm{O}_{7}$ $0,3 \mathrm{~mol} / \mathrm{L}$, utilizado no banho como agente complexante do Cobalto. Neste caso, a concentração de $\mathrm{CoSO}_{4}$ variou de 0,1 a 0,3 $\mathrm{mol} / \mathrm{L}$. Outros reagentes foram utilizados para fins específicos, como por exemplo: 1dodecilsulfato-Na 1,04.10-4 mol/L, para que o $\mathrm{H}_{2}$ liberado durante a eletrodeposição se desprenda com maior velocidade evitando a formação de bolhas na liga aderida e $\left(\mathrm{NH}_{4}\right)_{2} \mathrm{SO}_{4}$ 1,287.10-1 mol/L, para dar maior estabilidade ao banho. 
Para a análise de composição do banho diagramas de especiação dos componentes em função do $\mathrm{pH}$ foram simulados usando os programas Hydra e Medusa (PUIGDOMENECH, 2004) para se conhecer as diferentes espécies em meio aquoso. Esses diagramas foram construídos nas concentrações máximas e mínimas de espécies químicas iônicas nos ensaios de eletrodeposição. A especiação foi realizada considerando as razões estequiométricas dos sais dos metais utilizados.

O substrato utilizado na eletrodeposição foi uma placa de cobre na forma quadrada com $2 \mathrm{~cm}$ de lado. Esta foi inicialmente polida e, em seguida imersa em soluções $\mathrm{NaOH} 10 \%$, para realizar o desengraxe e $\mathrm{H}_{2} \mathrm{SO}_{4} 1 \%$ para ativação da superfície. Os ensaios foram realizados utilizando um eletrodo rotatório, um potenciostato/galvanostato, para controle da diferença de potencial elétrico entre o eletrodo de trabalho e o contraeletrodo, um banho termostático, para controle da temperatura e um medidor de $\mathrm{pH}$. A densidade de corrente elétrica variou de 10 a $50 \mathrm{~mA} / \mathrm{cm}^{2}$ e a temperatura entre 25 e $60{ }^{\circ} \mathrm{C}$. As eletrodeposições foram realizadas usando rotação catódica de $30 \mathrm{rpm}$. O potenciostato foi usado de forma galvanostática e o $\mathrm{pH}$ do banho foi aproximadamente 6.0 contraeletrodo utilizado foi uma malha cilíndrica oca de platina conforme a Figura 1.

Figura 1 - Esquema da célula de eletrodeposição

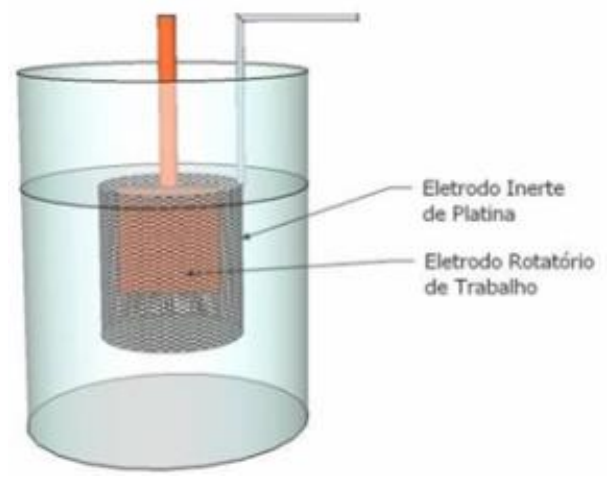

Para otimização do processo de eletrodeposição da liga de Co-W, foi realizado um planejamento fatorial $2^{3}$, com 3 experimentos no ponto central totalizando 11 experimentos (BRUNS et al., 1995). Analisou-se a influência da concentração inicial de Cobalto no banho $\left(\mathrm{C}_{\mathrm{Co}}\right)$, densidade de corrente elétrica (I) e temperatura do banho (T) sobre rendimento farádico $(\varepsilon)$.

Os experimentos foram realizados em ordem aleatória, para evitar o erro sistemático, as análises de regressão dos dados experimentais foram interpretadas utilizandose o software Statistica 5.0 para estimar o rendimento farádico a partir das variáveis de entrada.

\subsection{Eficiência de deposição.}

A eficiência de deposição é uma grandeza que quantifica a carga elétrica utilizada para a realização da eletrólise. No caso das ligas de $\mathrm{Co}-\mathrm{W}$, a eficiência de deposição deve ser calculada a partir das Equações 3 e 4:

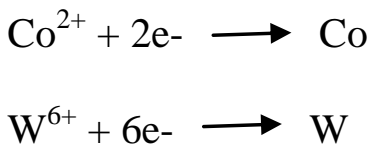

Para as ligas de Co-W em questão, a proporção mássica de $\mathrm{Co}-\mathrm{W}$ foi determinada por EDX. Isto faz com que parte ponderada da massa eletrodepositada seja de Co e a outra de W. Assim, as cargas elétricas foram obtidas pelas Equações 5 e 6 :

$$
\begin{gathered}
Q_{C o}=\frac{2 \cdot m_{C o} \cdot F}{M_{C o}} \\
Q_{W}=\frac{6 \cdot m_{W} \cdot F}{M_{W}}
\end{gathered}
$$

sendo:

- Q Co é a carga elétrica necessária para eletrodeposição de Co na liga, em Coulombs;

- $\mathrm{m}_{\mathrm{Co}}$ é a massa de Co depositada sobre o substrato de cobre, em gramas $(\mathrm{g})$;

- $\mathrm{M}_{\mathrm{Co}}$ é a massa atômica do Co, igual a $58,93 \mathrm{~g} / \mathrm{mol}$; 
- F é a constante de Faraday, igual a 96.485,34 Coulomb/mol;

- Qw é a carga elétrica necessária para eletrodeposição de W na liga, em Coulombs;

- $\mathrm{m}_{\mathrm{W}}$ é a massa de $\mathrm{W}$ depositada sobre o substrato de cobre, em gramas (g);

- $\mathrm{M}_{\mathrm{W}}$ é a massa atômica do $\mathrm{W}$, igual a $183,85 \mathrm{~g} / \mathrm{mol}$.

Somando-se as Equações 5 e 6, obtémse a Equação 7:

$\mathrm{Qu}=\mathrm{Q}_{\mathrm{Co}}+\mathrm{Q}_{\mathrm{W}}$

em que Qu, dada em Coulombs, representa a carga elétrica utilizada para a eletrodeposição de determinada quantidade mássica de liga Co-W.

A carga elétrica total presente no sistema é dada pela Equação 8, expressa por:

$\mathrm{Q}=\mathrm{i} \cdot \mathrm{t}$

sendo:

- Q é a carga elétrica total presente no sistema, dada em Coulombs;

- i é a intensidade da corrente elétrica, dada em Ampères (A);

- t é o tempo, dado em segundos (s).

A razão entre a carga elétrica utilizada e a carga total presente no sistema fornece a eficiência de deposição do processo, dada pela Equação 8:

$\varepsilon=\frac{Q_{u}}{Q} \cdot 100$

sendo, $\varepsilon$ dada em termos percentuais (\%) e conhecida também como "rendimento farádico".

\subsection{Caracterização}

As amostras da liga de Co-W foram caracterizadas por Microscopia Eletrônica de Varredura com Análise Química por Energia Dispersiva de Raios X (EDX). As micrografias dos substratos de cobre contendo as ligas metálicas de tungstênio foram obtidas no microscópio eletrônico de varredura (MEV) da marca LEO, modelo LEO 440i com detector de EDX.

\section{RESULTADOS E DISCUSSÃO}

\subsection{Especiação}

$\mathrm{O} \mathrm{pH}$ do banho influencia o potencial da descarga do hidrogênio e a composição dos complexos com os metais a serem depositados. Esses complexos dependem das condições de $\mathrm{pH}$, concentração e força iônica para se estabelecerem. Neste contexto, os diagramas químicos de especiação metálicas, possuem o objetivo de determinar o $\mathrm{pH}$ dos banhos eletrolíticos para obtenção da liga CoW. O pH dos banhos eletrolíticos afeta o processo de eletrodeposição, bem como determinam as diferentes espécies metálicas que podem existir nas soluções aquosas usadas durante as eletrólises.

Figura 2 - Espécies de cobalto no banho eletrolítico

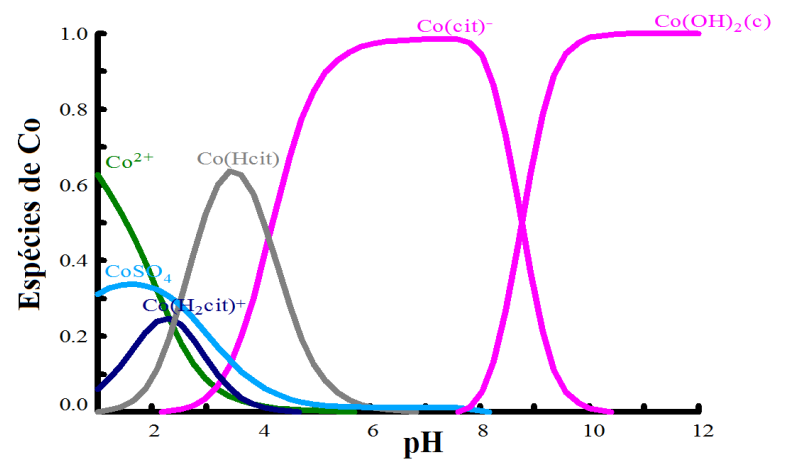

Figura 3 - Espécies de tungstênio no banho eletrolítico

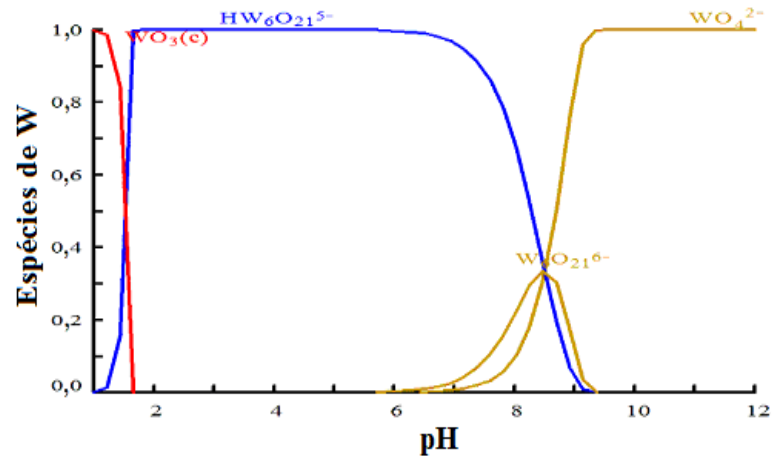


De acordo com os diagramas de especiação da Figura 2 é possível observar que, as espécies químicas $\mathrm{Co}^{2+}$ são complexadas pelo citrato de amônio na faixa de $\mathrm{pH}$ que vai de 5 a 8 . Por outro lado, na Figura 3 as espécies químicas de $\mathrm{W}$ que surgem na faixa de $\mathrm{pH}$ em que o cobalto é complexado ( $\mathrm{pH}=5-8)$ são o meta-tungstato ácido e o meta-tungstato (PRASAD, 2007). Com base nesses dados, utilizou-se o banho eletrolítico com o pH apresentado após a sua preparação, sendo este valor em torno de 6 .

\subsection{Análises Químicas}

A análise por Energia Dispersiva de Raios X (EDX) nos fornece uma composição química qualitativa e semiquantitativa dos metais presentes nos filmes bem como da homogeneidade dos mesmos quanto a sua composição. A partir do conhecimento da composição dos metais presentes no filme foi possível calcular a quantidade de massa depositada de cada metal e posteriormente a eficiência de deposição em cada experimento.

As análises de EDX foram realizadas no centro e nas bordas das amostras, para investigar a homogeneidade do sistema, conforme dados da Tabela 1.

Tabela 1 - Porcentagens dos metais presentes em diferentes regiões dos substratos

\begin{tabular}{ccccc}
\hline & \multicolumn{2}{c}{ Centro } & \multicolumn{2}{c}{ Borda } \\
\cline { 2 - 5 } Exp. & Co $(\%)$ & W $(\%)$ & Co $(\%)$ & W $(\%)$ \\
\hline 1 & 69,26 & 31,3 & 69,65 & 30,35 \\
2 & 81,91 & 18,09 & 84,43 & 17,57 \\
3 & 56,74 & 43,26 & 59,22 & 40,78 \\
4 & 80,25 & 19,21 & 82,58 & 17,03 \\
5 & 68,19 & 28,73 & 69,17 & 27,74 \\
6 & 78,34 & 21,67 & 79,69 & 20,3 \\
7 & 55,45 & 42,78 & 57,91 & 40,32 \\
8 & 71,13 & 27,80 & 80,25 & 18,25 \\
9 & 68,70 & 30,12 & 70,25 & 28,25 \\
10 & 64,08 & 34,87 & 70,88 & 27,76 \\
11 & 68,7 & 30,3 & 69,03 & 29,86 \\
\hline
\end{tabular}

Pode-se observar, a partir da Tabela 1, comparando a porcentagem dos metais presentes no centro da amostra com a porcentagem dos metais presentes na borda, uma mesma tendência na composição dos metais depositados, ou seja, quando o Co esta presente em maior quantidade no centro, também se apresenta em maior quantidade na borda. Pode-se considerar que de modo geral todas as amostras são homogêneas, ou seja, os materiais se mostram uniformes em sua composição, o que indica uma distribuição de corrente por toda superfície do eletrodo, não ocorrendo efeito de borda.

Com o conhecimento das porcentagens em massa dos metais depositados no substrato de cobre foi possível realizar o cálculo de eficiência de deposição, baseando-se nas massas de Co e $\mathrm{W}$ em cada liga metálica, como apresentado na Tabela 2.

Tabela 2 - Massas de Co e W presentes nas ligas

\begin{tabular}{ccc}
\hline Exp. & $\mathrm{m}_{\mathrm{Co}}(\mathrm{g})$ & $\mathrm{m}_{\mathrm{W}}(\mathrm{g})$ \\
\hline 1 & 0,0445 & 0,01976 \\
2 & 0,0573 & 0,0132 \\
3 & 0,1153 & 0,0835 \\
4 & 0,2991 & 0,0649 \\
5 & 0,0394 & 0,01598 \\
6 & 0,0564 & 0,0149 \\
7 & 0,1335 & 0,0972 \\
8 & 0,2789 & 0,0773 \\
9 & 0,1432 & 0,0569 \\
10 & 0,1552 & 0,0668 \\
11 & 0,1555 & 0,0633 \\
\hline
\end{tabular}

\subsection{Planejamento experimental.}

A Tabela 3 apresenta as variáveis utilizadas no planejamento fatorial, suas codificações e os níveis reais de cada variável.

Tabela 3 - Valores reais e codificados das variáveis do planejamento experimental $2^{3}$

\begin{tabular}{lccc}
\hline & $(-1)$ & 0 & $(+1)$ \\
Variáveis & & & \\
\hline $\mathrm{C}_{\mathrm{Co}}(\mathrm{mol} / \mathrm{L})$ & 0,1 & 0,2 & 0,3 \\
$\mathrm{I}\left(\mathrm{mA} / \mathrm{cm}^{2}\right)$ & 10 & 30 & 50 \\
$\mathrm{~T}\left({ }^{\circ} \mathrm{C}\right)$ & 25 & 42,5 & 60 \\
\hline
\end{tabular}

Na Tabela 4 está apresentada a matriz do planejamento utilizado no processo de 
otimização dos parâmetros do banho do sistema de eletrodeposição.

Tabela 4 - Matriz do planejamento fatorial $2^{3}$ da liga $\mathrm{Co}-\mathrm{W}$

\begin{tabular}{ccccc}
\hline Exp. & $\begin{array}{c}\mathrm{C}_{\mathrm{Co}} \\
(\mathrm{mol} / \mathrm{L})\end{array}$ & $\begin{array}{c}\mathrm{I} \\
\left(\mathrm{mA} / \mathrm{cm}^{2}\right)\end{array}$ & $\begin{array}{c}\mathrm{T} \\
\left({ }^{\circ} \mathrm{C}\right)\end{array}$ & $\varepsilon(\%)$ \\
\hline 1 & -1 & -1 & -1 & 72,19 \\
$\mathbf{2}$ & $\mathbf{1}$ & $\mathbf{- 1}$ & $\mathbf{- 1}$ & $\mathbf{8 6 , 6 1}$ \\
3 & -1 & 1 & -1 & 44,47 \\
4 & 1 & 1 & -1 & 82,20 \\
5 & -1 & -1 & 1 & 62,26 \\
6 & 1 & -1 & 1 & 82,15 \\
7 & -1 & 1 & 1 & 51,61 \\
8 & 1 & 1 & 1 & 80,32 \\
9 & 0 & 0 & 0 & 75,01 \\
10 & 0 & 0 & 0 & 83,16 \\
11 & 0 & 0 & 0 & 82,00 \\
\hline
\end{tabular}

A análise de variância (ANOVA) foi realizada com um nível de confiança de $95 \%$ para $\mathrm{p}<0,05$. Os resultados da ANOVA listados na Tabela 5 demonstram que 0 modelo estatístico é significante e preditivo para $\mathrm{p}<0,05$.

Verificou-se que a concentração de sulfato de cobalto foi a variável de entrada que mais influenciou estatisticamente $o$ processo.

Tabela 5 - Resultados da ANOVA para eficiência de deposição

\begin{tabular}{lcccc}
\hline \multicolumn{1}{c}{ Fontes } & $\begin{array}{c}\text { Soma } \\
\text { quadrática }\end{array}$ & $\begin{array}{c}\text { Grau de } \\
\text { liberdade }\end{array}$ & $\mathrm{T}$ & $\mathrm{P}$ \\
\hline $\mathrm{C}_{\mathrm{Co}}(1)$ & 41,49 & 1 & 4,28 & 0,012 \\
$\mathrm{I}(2)$ & 5,15 & 1 & $-1,89$ & 0,130 \\
$\mathrm{~T}(3)$ & 14,02 & 1 & $-0,38$ & 0,717 \\
$\begin{array}{l}\text { Interação } \\
1 \text { e 2 }\end{array}$ & 24,33 & 1 & 1,36 & 0,243 \\
$\begin{array}{l}\text { Interação } \\
1 \text { e 3 }\end{array}$ & 15,41 & 1 & $-0,15$ & 0,887 \\
$\begin{array}{l}\text { Interação } \\
\text { 2 e 3 }\end{array}$ & 21,21 & 1 & 0,83 & 0,449 \\
\hline
\end{tabular}

\subsection{Efeito da concentração de $\mathrm{CoSO}_{4}$}

O estudo do efeito da concentração de sulfato de cobalto com relação à eficiência de deposição foi avaliado para a faixa de concentrações apresentadas na Tabela 3. Na Figura 4 está apresentado o comportamento da eficiência de deposição com relação às concentrações de $\mathrm{CoSO}_{4}$ e temperaturas avaliadas. Foi observado que com o aumento da concentração do $\mathrm{CoSO}_{4}$ foram obtidos os maiores valores de eficiência de deposição (Tabela 4). O valor otimizado encontrado foi de $0,3 \mathrm{~mol} / \mathrm{L}$.

Figura 4 - Superfície de resposta para eficiência de deposição de Co-W em função da concentração de $\mathrm{CoSO}_{4}$ e da temperatura em $\mathrm{pH} 6$

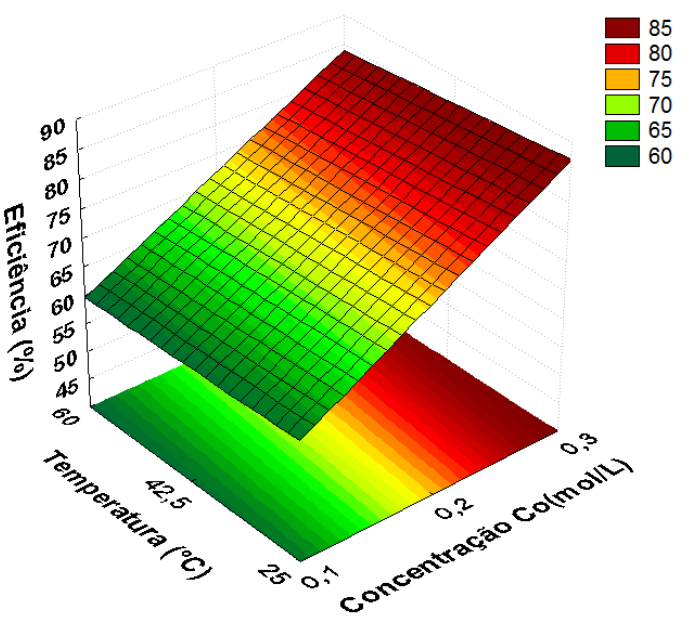

\subsection{Análise morfológica das ligas de Co-W}

As Figuras 5-9 mostram as micrografias obtidas no MEV para os revestimentos de Co-W. Observou-se que as morfologias das camadas apresentaram nódulos e certa uniformidade. Os nódulos esféricos na extensão da superfície e apresentaram uniformidade em termos de tamanho. Pode-se observar que na Figura 5, experimento 10, a liga possui trincas na superfície da camada de $\mathrm{Co}-\mathrm{W}$.

Como os átomos de $\mathrm{W}$ possuem raios atômicos maiores que os átomos de cobalto, isto gera deformações no retículo, que durante o processo de eletrodeposição promovem a formação de trincas. As trincas também podem estar associadas à tensão superficial entre os metais presentes na liga, o que pode comprometer seu desempenho de proteção contra corrosão, pois as trincas permitirão a passagem do eletrólito até o substrato. 
Figura 5 - Micrografia do revestimento de Co-W. Experimento 10, concentração de $\mathrm{CoSO}_{4} \quad 0,2$ $\mathrm{mol} / \mathrm{L}$, temperatura $42,5^{\circ} \mathrm{C}$ e densidade de corrente $30 \mathrm{~mA} / \mathrm{cm}^{2}$

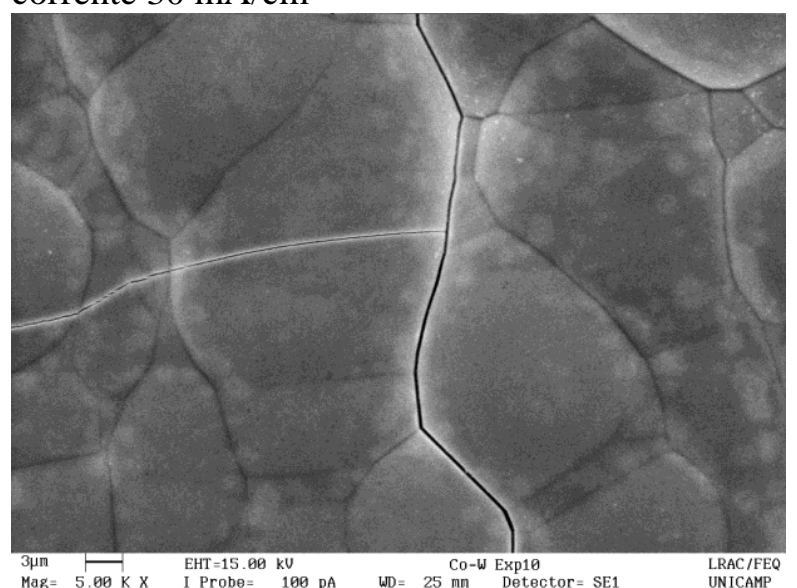

Observa-se na Figura 6 que o revestimento de $\mathrm{Co}-\mathrm{W}$ apresenta uma tendência de uniformidade na liga com poucos nódulos na superfície.

Figura 6 - Micrografia da liga de Co-W. Experimento 11, concentração de $\mathrm{CoSO}_{4} \quad 0,2$ $\mathrm{mol} / \mathrm{L}$, temperatura $42,5^{\circ} \mathrm{C}$ e densidade de corrente $30 \mathrm{~mA} / \mathrm{cm}^{2}$

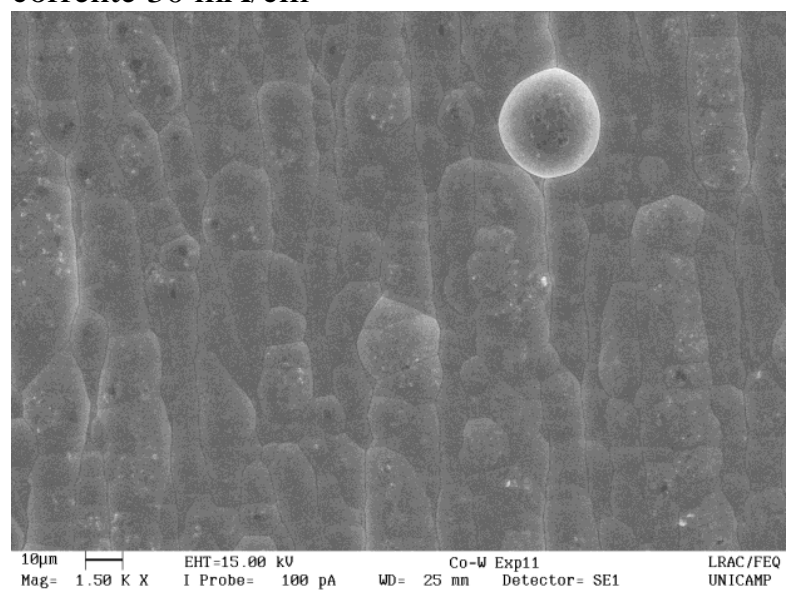

Comparando as Figuras 7 e 8 correspondentes respectivamente os experimentos 4 e 7 , pode ser observado que revestimentos com maior quantidade de nódulos apresentam uma tendência a superposição de camadas com a formação de uma primeira camada sobre o substrato e uma codeposiçao sobre a camada Co-W, com a formação de nódulos isolados que vão coalescendo e formando uma segunda camada.

Figura 7 - Micrografia de Co-W. Experimento 4, concentração de $\mathrm{CoSO}_{4} 0,3 \mathrm{~mol} / \mathrm{L}$, temperatura $60^{\circ} \mathrm{C}$ e densidade de corrente $10 \mathrm{~mA} / \mathrm{cm}^{2}$

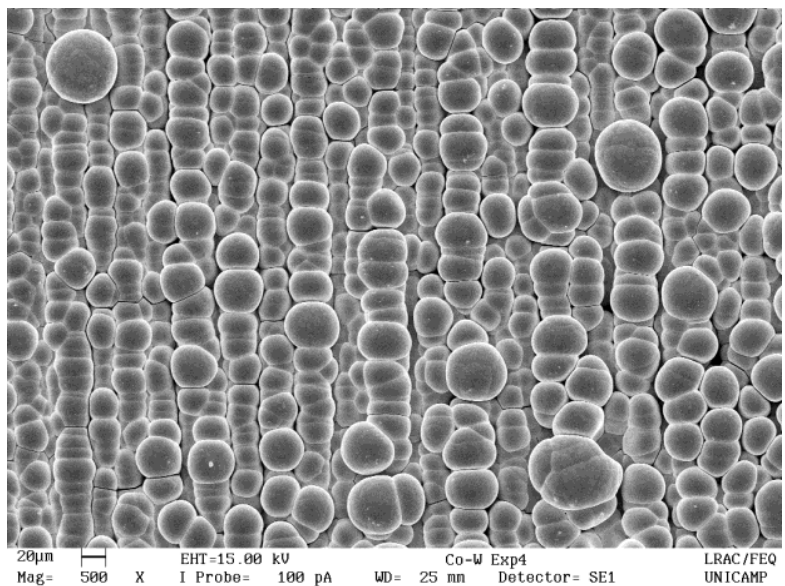

Figura 8 - Micrografia dos eletrodepositos de Co-W. Experimento 7, concentração de $\mathrm{CoSO}_{4} 0,3$ $\mathrm{mol} / \mathrm{L}$, temperatura $25^{\circ} \mathrm{C}$ e densidade de corrente $30 \mathrm{~mA} / \mathrm{cm}^{2}$

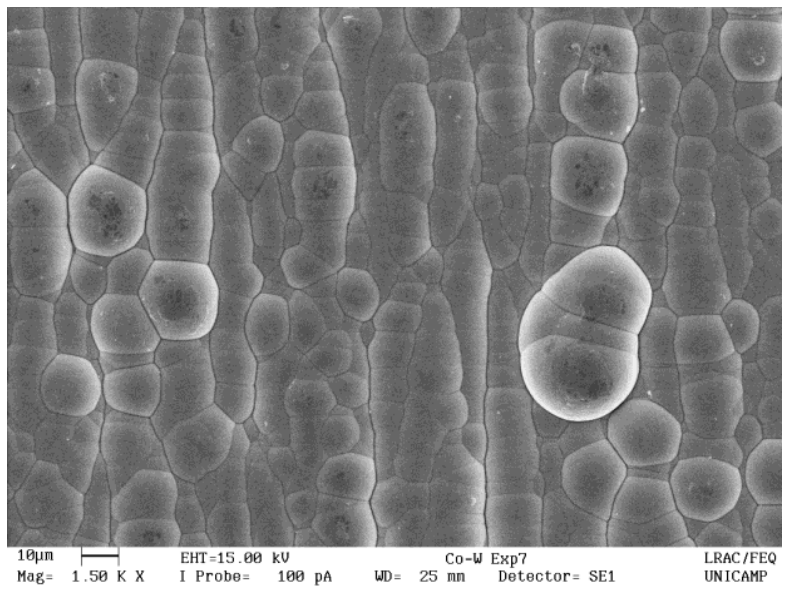

Observa-se ainda que o revestimento apresentado na Figura 9 correspondente ao experimento 2, além de ter obtido a maior eficiência de deposição também não apresentou trincas superficiais. Nesse caso, o revestimento foi homogêneo, compacto e com boa uniformidade. 
Figura 9 - Micrografia do experimento 2, concentração de $\mathrm{CoSO}_{4} 0,3 \mathrm{~mol} / \mathrm{L}$, temperatura a $25^{\circ} \mathrm{C}$ e densidade de corrente igual a $10 \mathrm{~mA} / \mathrm{cm}^{2}$

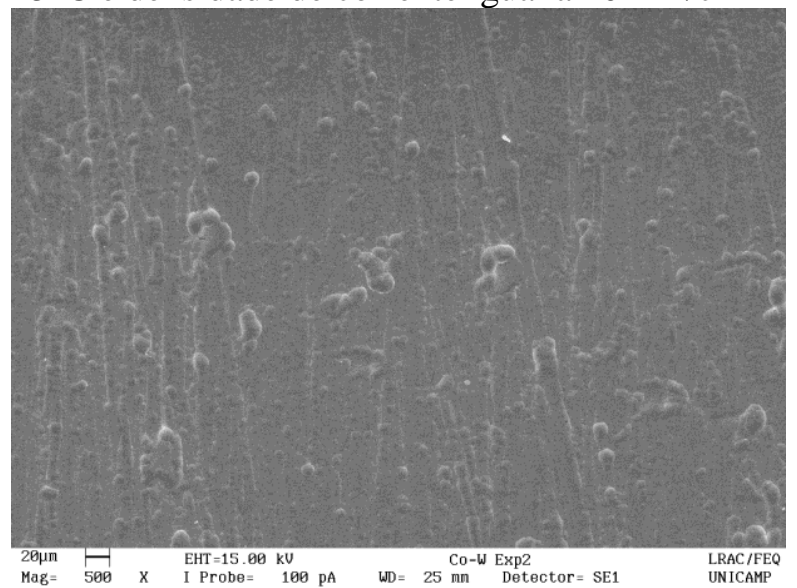

\section{CONCLUSÃO}

Os resultados mostram que dentro da faixa de variáveis propostas, a maior porcentagem de deposição encontrada foi de $86,61 \%$ do experimento 2, utilizando concentração de sulfato de cobalto $0,3 \mathrm{~mol} / \mathrm{L}$, temperatura a $25^{\circ} \mathrm{C}$ e densidade de corrente igual a $10 \mathrm{~mA} / \mathrm{cm}^{2}$. As ligas obtidas apresentaram boa aderência.

\section{REFERÊNCIAS}

AZEVEDO, C. A. C; WORMSBECKER, L. E.; ADAIME, L. M.; MILEK, J. L.; Scheer, s. Corrosão em estruturas metálicas. Disponível em: <http://www.cesec.ufpr.br/metalica/patologi as/corrosao.htm>. Acesso em: 29 maio. 2015.

BRENNER, A. Eletrodeposition of alloys Principles and Practice. v.1 e 2. New York: Academic Press, 1964.

DELAHAY, P. Double layer and electrode Kinetics. New York: Wiley Interscience, 1965.

GENTIL, V. Corrosão, Rio de Janeiro: Livros Técnicos e Cientificos Editora S.A, 1996.
KOTZ, J. C.; TREICHEL, P. M. Química Geral 2 e Reações Químicas. São Paulo: Thomson Learning Edições, 2005.

PRASAD, S.; SANTANA, R. A. C; CAMPOS, A. R. N; LEITE,V. D. Potentiometric and conductometric studies on the system acid-isopolytungstate and the formation of lanthanum tungstates. Eclética Química, v.32, p.71-78, 2007.

PUIGDOMENECH, P. Chemical Equilibrium Diagrams. Stockholm, Sweden: Inorganic Chemistry Royal Institute of Technology (KTH), 2004.

WOLYNEC, S. Corrosão de metais: Definição e principais tipos. Anais do Simpósio de Materiais de Construção: Durabilidade dos materiais e componentes de construção civil. São Paulo, EP/USP, 1998. p.15-31.

\section{AGRADECIMENTOS}

Os autores agradecem ao FAEPEX/UNICAMP e à FAPESP pelo apoio financeiro. 